\title{
Discussão sobre algumas contribuições da modelagem multinível para a investigação de desempenho no trabalho
}

\author{
Francisco Antonio Coelho Junior - Universidade de Brasilia, Brasilia, Brasil \\ Jairo Eduardo Borges-Andrade - Universidade de Brasilia, Brasilia, Brasil
}

\begin{abstract}
Resumo
O investimento no capital intelectual tornou-se o pilar norteador das ações de gerenciamento de pessoas. A literatura em comportamento organizacional investigada enfatiza o efeito de variáveis de contexto no desempenho dos indivíduos. Fazse mister compreender como se dá o impacto dessas variáveis segundo seus níveis de análise. O presente artigo discute contribuições potenciais da modelagem multinível na investigação de desempenho no trabalho. Discute, ainda, a aplicação dessa modelagem para compreensão de fenômenos comumente investigados em comportamento organizacional. Tais contribuições poderão favorecer a estruturação de modelos preditivos que poderão melhor capturar o significado da inclusão de variáveis típicas do contexto laboral.

Palavras-chave: Modelagem multinível, Desempenho no trabalho, Comportamento organizacional.
\end{abstract}

\section{Discussing some multilevel models contributions to investigate performance at work}

\begin{abstract}
Investing on intellectual capital became the guiding pillar for personnel management actions. The organizational behavior literature highlights the effect of contextual variables on the performance of individuals. The impact of these variables, which belong to different levels of analysis, needs a better comprehension. This paper discusses the potential multilevel modeling contributions for investigating predictors of that performance. In addition, it also discusses the application of this modeling for understanding usually studied organizational behavior. These contributions may favor structuring predictive models which may better grasp the meaning of the inclusion of diverse and complex levels of variables from the labor context.
\end{abstract}

Keywords: Multilevel models, Performance at work, Organizational behavior.

Os indivíduos, em situação de trabalho, podem ou não desejar realizar atividades produtivas ou utilizar o que sabem para melhorá-las. As razões para isso podem, por exemplo, estar relacionadas aos valores dessas pessoas, ao suporte social e organizacional que percebem e ao que acreditam que é delas esperado. Todas essas razões são geralmente verificadas no nível individual, mesmo que façam referência à crença sobre fenômenos do contexto, por exemplo. Para que este contexto possa ser efetivamente considerado como associado ao desempenho do indivíduo, ele precisa ser definido e medido um nível "acima" do indivíduo, seja este nível a equipe de trabalho, seja o setor de trabalho, a unidade organizacional ou diferentes organizações. A modelagem multinível, então, foi proposta como estratégia para considerar tais situações.

A modelagem multinível vem sendo comumente empregada nos estudos científicos relacionados à educação, marketing e saúde. Poucos estudos, porém, foram desenvolvidos em comportamento organizacional aplicando pressupostos desse tipo de modelagem, ainda mais a aplicando à

${ }^{1}$ Endereço para correspondência:

Universidade de Brasília - Faculdade de Economia, Administração, Contabilidade e Ciência da Informação e Documentação - Campus Universitário Darcy Ribeiro.

Departamento de Administração - Sala B1-576 70910-900 - Brasília-DF, Brasil.

E-mail: acoelho@unb.br investigação do desempenho no trabalho. O presente ensaio teórico objetiva discutir a aplicação da modelagem multinível na investigação de desempenho individual no trabalho. Propõe-se, assim, debater aspectos teóricos e metodológicos considerando-se o nível de análise das variáveis antecedentes associadas a desempenho, ou o quanto de sua variância pode ser predita por variáveis pertencentes a distintos níveis de análise. Serão apresentados, ainda, com exemplos ilustrativos, os passos necessários à realização de uma análise multinível, conforme recomendação de Hox (2002).

\section{Revisão de literatura}

A literatura em desempenho no trabalho é ainda escassa no que se refere à utilização da abordagem multinível (Burke, Sapyr, Tesluk \& SmithCrowe, 2002). Por mais que seja sugerido que algumas variáveis de nível de contexto são capazes de impactar no desempenho dos indivíduos, a literatura empírica que efetivamente investiga estas relações é restrita, especialmente lançando mão desta abordagem.

Considera-se, assim, premente a necessidade de incorporar, efetivamente, a modelagem multinível nos desenhos de pesquisa em comportamento organizacional, de modo a consolidá-la como importante instrumento de planejamento, análise e interpretação de pesquisas empíricas relacionadas a desempenho individual no contexto das organizações. Desempenho pode ser considerado como um 
construto de natureza multideterminada, isto é, associado a fatores de nível individual (querer fazer), relacionados à tarefa (saber fazer) e ao contexto (poder fazer). A modelagem multinível pode ser apropriada para a investigação de desempenho individual no trabalho, posto que considera o nível de análise de cada variável por meio de seus pressupostos básicos e permite a investigação, também, do quanto da parcela de variabilidade de desempenho pode ser explicada por variáveis oriundas de níveis distintos. Isto é importante, visto que o desempenho, multicausal, é dependente de uma série de variáveis típicas de níveis distintos de análise, de nível menos elevado, como características pessoais, ou mais elevado, como o contexto.

Alguns poucos modelos empíricos brasileiros (como Brandão, 2008; Coelho Jr., 2009) já podem ser encontrados na literatura de comportamento organizacional fazendo referência à aplicação da modelagem multinível na compreensão de desempenho no trabalho. As características antecedentes relacionadas à investigação de desempenho no trabalho não têm, historicamente, variado de nível de análise (Sonnentag \& Frese, 2002). A investigação dessas variáveis, bem como a interação entre elas, pode facilitar a compreensão sobre o desempenho no trabalho e, portanto, possibilitar sua gestão.

Boa parte das inferências realizadas em artigos empíricos podem ter sido feitas de maneira limitada, sem considerar, efetivamente, o nível de análise ao qual cada variável realmente pertence. A falta de alinhamento dos itens das medidas em relação aos construtos associados pode ocasionar erros de inferência ou de generalização dos resultados.

Quando se investiga, por exemplo, cultura organizacional, espera-se que o teor dos itens que comporão a sua medida remeta a características organizacionais, a fim de que haja precisão da avaliação em relação ao construto associado. Ou seja, que o nível de análise dos itens compreenda aspectos de contexto. Essa simples operacionalização de uma suposição teórica é fundamental à compreensão de diversos fenômenos estudados em comportamento organizacional. Parte-se da pressuposição básica de que fenômenos devem ser analisados corretamente em seu nível. Fenômenos contextuais também devem ser compreendidos como tal. A construção das medidas e instrumentos de pesquisa deve sempre levar em conta a natureza do construto analisado.

A pretensão aqui não é simplificar toda a complexidade da utilização da modelagem multinível aplicada a estudos organizacionais. Todavia, o estado da arte já permite discutir a implicação desta modelagem para compreensão de desempenho humano no trabalho. $\mathrm{O}$ uso desse tipo de modelagem em estudos sobre comportamento organizacional precisa se consolidar como importante agenda de pesquisa.

\section{Desempenho no trabalho}

Variáveis de contexto, como resultados organizacionais ou indicadores macro de eficácia, são comumente relacionadas a desempenho manifestado no trabalho (Nielsen, 2006). Tais variáveis predizem desempenho em conjunto com outras variáveis, de ordem individual ou mesmo profissional. Os estudos em que desempenho no trabalho é investigado como variável critério usualmente lançam mão de uma abordagem individual de investigação das variáveis antecedentes (Sonnentag \& Frese, 2002). Isso ocorre em virtude das organizações se preocuparem em melhorar o desempenho dos indivíduos a partir do reconhecimento de suas dificuldades, não considerando outros fatores de natureza macro, como o contexto de trabalho e a influência deste sobre o desenho do cargo (Lam \& Schaubroek, 1999).

Adota-se aqui a definição de Sonnentag e Frese (2002), de que desempenho refere-se a habilidades e competências aplicadas no contexto do cargo à execução de uma obrigação ou tarefa, ou à maneira como atua um indivíduo em termos de eficiência e rendimento, resultando no alcance de resultados organizacionais. Refere-se ao empreendimento intencional pelo indivíduo em uma ação que é orientada pelo seu resultado, ou seja, uma ação que tem um propósito consciente ou motivação prévia.

Desempenho está condicionado a um conjunto de fatores relacionados ao contexto de trabalho do indivíduo (condições sociais, culturais e relativas ao posto de trabalho do indivíduo) e relaciona-se à qualidade e quantidade de trabalho realizado, bem como ao custo e tempo despendidos na sua execução. Abrange fatores de eficiência (meios ou processos) e eficácia (resultado efetivo) no âmbito da organização.

Este foco na análise de fatores individuais deve ser modificado, visando, fundamentalmente, abarcar variáveis críticas também dos níveis de grupos e equipes de trabalho, por exemplo. A carência de estudos na literatura sobre comportamento organizacional investigando relações de causa e efeito entre variáveis pode ser justificada na dificuldade associada à diferenciação das causas sistêmicas do desempenho em relação às causas individuais, especialmente quando se analisa a literatura em psicologia organizacional e do trabalho e de administração (Nielsen, 2006). 
Há evidências empíricas que sugerem que variáveis de contexto são capazes de predizer desempenho (Brandão, 2008; Coelho Jr., 2009; Derue \& Morgeson, 2007; Morgeson \& Hoffmann, 1999). Contudo, poucos são os estudos organizacionais cuja variável critério seja desempenho individual, ainda mais associada a uma perspectiva multinível, como foi o caso de Coelho Jr. (2009).

\section{Modelagem Multinivel}

Agrupamentos sociais ou contextos de atuação profissional, como um departamento, por exemplo, influenciam as pessoas neles inseridos, mas tais grupos e suas propriedades são também influenciados por essas pessoas (Hox, 2002; Maas \& Hox, 2005). Como esses grupos ou sistemas podem ser observados em diferentes níveis hierárquicos e as variáveis podem ser definidas isoladamente em cada nível de análise segundo sua definição teórica constitutiva e decorrente operacionalização, indivíduos e grupos sociais são tratados conceitualmente como um sistema hierárquico, em que as propriedades desses grupos são influenciadas pelos indivíduos que fazem parte dos mesmos (Maas \& Hox, 2005).

Assim como sistemas podem ser interpretados sob a ótica de diferentes níveis (subsistemas ou componentes integrantes) e a análise do todo é sempre mais do que a simples investigação particular das variáveis observadas em distintos níveis hierárquicos, algumas estratégias de interpretação existem para analisar dados em uma perspectiva multinível (Klein \& Kozlowski, 2000). Nesse caso, o desenho das pesquisas empíricas que lançam mão da modelagem multinível é planejado considerando o quanto da variância da variável critério será predito por meio do efeito de variáveis pertencentes a diferentes níveis de análise e o quanto esta diferenciação entre os níveis de análise impacta no melhor ajuste do modelo testado (Maas \& Hox, 2005). A especificação de construtos multiníveis deve estar alinhada ao método, possibilitando a composição e decomposição de variáveis que terão, ou não, efeitos significativos na predição de variância da variável critério associada (Bliese, Chan \& Ployart, 2007).

O modelo de regressão multinível pode ser considerado mais complexo em relação ao tradicional modelo de regressão múltipla, em virtude, especialmente, do número de parâmetros adotados, considerado maior nos modelos multiníveis (Hox, 2002). Segundo Hox, ainda, na abordagem multinível, os dados são agrupados e mais complexos para análise e interpretação do que os modelos de regressão tradicionais, já que se investiga a emergência de fenômenos de nível individual para os níveis de setor ou equipe e organizacional.

Algumas pesquisas brasileiras e internacionais foram desenvolvidas sistematicamente nos últimos anos, verificando-se o efeito da interação entre as variáveis que caracterizam o indivíduo e seu contexto de inserção ou de trabalho (Brandão, 2008; Coelho Jr., 2009; Krull \& MacKinnon, 1999; Lester, Meglino \& Korsgaard, 2002; Oliveira, 2007). Os modelos multiníveis mais utilizados são o de componentes de variância, em que apenas o intercepto é aleatório e a variância da variável dependente é decomposta entre níveis, e o de coeficientes aleatórios, que testa o efeito randômico dos coeficientes de inclinação. $\mathrm{O}$ passo a passo relativo à inserção de cada termo será apresentado a seguir, ilustrando os tipos de modelos multiníveis mais comumente encontrados na literatura disponível.

Isso significa afirmar que o modelo de regressão multinível incorpora naturalmente a estrutura hierárquica dos dados, tratando o intercepto e os coeficientes de inclinação como variáveis aleatórias, ou seja, assumindo-se que cada unidade do segundo nível pode ter o componente da variância. A título de exemplificação pode-se supor que departamentos em uma mesma organização podem ter subculturas organizacionais que lhe são próprias e que diferenciam o comportamento dos indivíduos durante a execução de suas tarefas. Tal variabilidade é determinada por fatores de nível macro (como crenças e valores coletivamente compartilhados) e é capaz de impactar em variáveis de nível micro, tais como satisfação no trabalho e desempenho individual.

Se há efeitos do contexto social sobre o comportamento de indivíduos, esses efeitos devem ser analisados considerando-se os processos intervenientes que dependem das características desse ambiente (Maas \& Hox, 2005). Isso significa afirmar que variáveis de nível diferenciado podem agir isoladamente ou em interação de maneira a produzir efeitos específicos, considerando-se o contexto que está sendo analisado.

A variável resposta ou critério em um modelo de regressão multinível é sempre uma variável de nível menos elevado. As variáveis explicativas ou antecedentes podem pertencer a distintos níveis, quer sejam individuais, quer sejam grupais ou de nível macro ou organizacional, como indicadores financeiros de performance, competências organizacionais ou cultura organizacional, por exemplo. A adoção de um modelo multinível parte da pressuposição básica de que, em relação à variável critério, existem diferenças tanto do nível mais baixo quanto do nível mais elevado, independentemente do fenômeno de investigação representado (Maas \& Hox, 2005). Ao se investigar 
desempenho nas organizações como variável critério, por exemplo, considera-se que o escore obtido pelo indivíduo pode ser mais bem interpretado caso se considerem variáveis pertencentes a ele e ao seu contexto de trabalho, assumindo-se que esse escore pode variar de funcionário para funcionário, ou seja, há variância no nível individual, assim como de uma unidade organizacional para outra (há variância no nível de contexto). O primeiro tipo de variância é conhecido como "intranível", entre elementos do nível mais baixo; o segundo tipo, "entrenível", quando são incorporadas variâncias de nível de contexto, calculando-se, então, a variância total que for significativa em todos os níveis contemplados no modelo teórico hipotetizado. Os métodos de estimação comumente utilizados em análises multiníveis (como o maximoverossimilhança, ML) são assintóticos, partindo da premissa fundamental de que o tamanho da amostra deve ser suficientemente grande. Isso traz questões referentes ao limite mínimo aceitável em relação ao tamanho da amostra, de modo que a acurácia da estimação e dos erros padrões associados é mais frágil em amostras pequenas, que se tornam mais suscetíveis na generalização dos resultados. Em estudos multiníveis, o problema central encontra-se no tamanho da amostra no nível de grupos, porque esse tamanho é sempre menor que o tamanho da amostra no nível mais inferior (Afshartous \& Leeuw, 2005; Maas \& Hox, 2005).

As percepções individuais de um mesmo grupo, quando convergem, são tratadas como um prérequisito fundamental à agregação das variáveis de nível mais baixo para os níveis mais elevados. Dessa forma, a análise das medidas de dispersão é utilizada para identificar a variabilidade entre características individuais e verificar como se dá a contribuição individual para a formação de um dado grupo. Devese, inclusive, calcular o grau de correlação intraclasse, além dos coeficientes de variação e medidas de dispersão associadas, a fim de justificar a investigação de uma dada variável de nível mais baixo em um nível mais elevado (Roberson, Sturman \& Simons, 2007).

Algumas vantagens da utilização de modelos multiníveis em relação aos modelos clássicos de regressão são apresentadas por Oliveira (2007). Elas dizem respeito a estimativas dos parâmetros providos, bem como à testagem de hipóteses relativas aos efeitos de variáveis entre níveis e análise da magnitude da variância por meio da variância compartilhada. Tem-se, assim, informações mais precisas acerca dos parâmetros estimados e do grau de ajustamento do modelo testado, embora a variância em nível de grupos seja passível da influência de um sem-número de variáveis intervenientes à relação teórica hipotetizada.
A seguir, apresentam-se os procedimentos necessários à realização de uma análise multinível. Cada passo será exemplificado com variáveis tipicamente investigadas em estudos de comportamento organizacional atuando na predição de desempenho individual no trabalho.

Passo a passo da análise multinivel e sua predição de desempenho

Alguns passos devem ser seguidos na análise multinível, segundo propõe Hox (2002). O primeiro deles começa pelo cálculo do modelo vazio. com o qual, têm-se as estimativas iniciais de variância tanto do nível individual quanto do contextual. Deve-se proceder ao cálculo do coeficiente de correlação intraclasse (ICC), que se dá pela divisão da variância de nível de contexto pelo somatório das variâncias de nível individual e de contexto. O ICC avalia o quanto da variação total nos escores atribuídos ao escore de desempenho se deve a diferenças entre as unidades organizacionais pesquisadas (setores e departamentos, por exemplo). Segundo Hox (2002), o ICC pode variar de 0 a 1 . Quando seu valor é nulo ou se aproxima de zero, isso significa que as Unidades analisadas são homogêneas entre si e que o desempenho do individuo é independente da Unidade em que trabalha. Ou seja, o contexto não exerceria efeitos significativos sobre os resultados alcançados. O recomendado é que o ICC seja próximo de 1 (Hox, 2002), contudo, não existe um valor mínimo ou máximo ideal referendado na literatura.

O modelo vazio não possui qualquer variável explicativa adicionada e encontra-se representado na equação 1.

[1] Yij $=\gamma 00+B$ oj + eij

$\mathrm{Na}$ equação anterior o intercepto é representado por $\gamma 00$, Uoj corresponde ao residual no nível do grupo e eij ao residual do nível do indivíduo. Já o termo i remete ao nível de análise menos elevado, como o indivíduo, e o j à representação da mais elevada, ou de nível de contexto. Os dados referentes ao intercepto, no modelo vazio, podem ser interpretados como o valor esperado do desempenho do indivíduo sem considerar a influência de qualquer variável explicativa testada no modelo. Caso a variável critério seja desempenho, a performance do funcionário i que trabalha na Unidade $\mathrm{j}$ é estimada pelo intercepto $(\gamma 00)$ considerando-se os termos de erro padrão associados (eij).

No modelo vazio tem-se, ainda, a estimativa inicial do índice de desajuste do modelo (deviance), que servirá de parâmetro de comparação em relação ao passo seguinte. Contudo, o valor encontrado na deviance não é o único índice utilizado para se verificar o ajuste do modelo. Analisa-se o tamanho das diferenças encontradas entre o seu valor e o valor encontrado no 
modelo seguinte. Recomenda-se, a partir de análise da literatura, como convencionado por Bliese, Chan e Ployhart (2007), Brandão (2008) e Coelho Jr. (2009), adotar como significativa a contribuição de uma variável quando a proporção da diferença entre os deviances entre os modelos testados $\left(\chi^{2}\right)$ pelo número de parâmetros adicionados em relação ao modelo anterior for igual ou maior que 2 . $\mathrm{Na}$ próxima equação são inseridas variáveis explicativas de nível individual.

[2] $\mathrm{Yij}_{\mathrm{ij}}=\gamma 00+\gamma \mathrm{p} 0+\mathrm{X} \mathrm{pij}+\mathrm{Uoj}+\mathrm{eij}$

Suponha-se que $\mathrm{O}$ pesquisador queira investigar o efeito de características individuais e profissionais sobre o desempenho manifestado pelo indivíduo. Nesta equação serão testadas $p$ variáveis explicativas de nível menos elevado (como idade, gênero, cargo e tempo de serviço). As variáveis individuais são inseridas, neste passo, com parâmetro fixo. Após a inserção de cada variável de nível individual, deve-se verificar se as mesmas são significativas, por meio do cálculo da razão crítica ou t, e se houve redução da deviance em relação à deviance obtida no modelo vazio. A deviance é um dos indicadores numéricos de ajustamento do modelo a partir da inserção de cada variável em cada passo adotado. O cálculo de significância da razão crítica é feito pelo Teste de Wald, que é utilizado para verificar se o parâmetro é estatisticamente significativo, e consiste na razão do coeficiente da variável pelo erro padrão associado. Esse valor deve ser superior a 1,96 (alguns autores utilizam o valor 2) para que a variável seja considerada significativa e possa contribuir para predição da variável critério associada. Caso contrário, ela não contribui de forma estatisticamente significativa para o modelo empírico. Para cada variável considerada estatisticamente significativa deve-se verificar, também, o componente de variância, seja individual, seja compartilhada, esperando-se que a variância diminua após a inserção de cada variável. Ressalta-se que, a cada modelo testado, espera-se que haja a redução da deviance, o que indica um melhor ajuste do modelo. Assim, o modelo anterior, de maior deviance, passa a ser descartado, e o modelo atual, de menor deviance, passa a ser referência para testagem dos modelos seguintes.

A equação seguinte, segundo Hox (2002), considera a inserção do termo zqj, que representa o conjunto de q variáveis explicativas de nível mais elevado. O termo $\gamma \mathrm{p} 0$ representa o coeficiente de inclinação fixado em zero, fixo. São inseridas aqui variáveis de nível de contexto, como crenças compartilhadas pelos indivíduos sobre algum fator (alguma característica da cultura organizacional, como o suporte à aprendizagem, que é provido por pares e chefias, por exemplo). Nota-se que, nesse passo, se nenhuma variável de nível de contexto contribuir de forma significativa, a modelagem multinível passa a não ser mais recomendada, posto que não há variabilidade significativa entreníveis que justificaria, assim, a sua utilização. A equação encontra-se representada a seguir.

[3] Yij $=\gamma 00+\gamma p 0 X p i j+\gamma p 0 Z q j+U o j+e i j$

Os passos 2 e 3, segundo Hox (2002), são conhecidos como modelos componentes de variância, haja vista que os mesmos decompõem a variância do intercepto em diferentes componentes de variância para cada nível hierárquico. O próximo passo (equação 4) consiste na inserção de termos randômicos na equação de regressão, conhecida como modelo de coeficientes randômicos. Diferentemente dos parâmetros fixos testados na equação 2 , afirma-se que o efeito exercido por uma variável é aleatório no nível 2 quando ele difere de Unidade (filiais, departamento ou setor, por exemplo) para Unidade. Os coeficientes de regressão fixos (equação 3) podem ser estimados mais precisamente do que os coeficientes randômicos (equação 4), já que o número de observações do nível menos elevado supera bastante o número de observações de nível de contexto (Hox, 2002). Os residuais das inclinações das variáveis explicativas de nível individual (X pij) são representados por Upj.

[4] Yij $=\gamma 00+\gamma p 0 X$ pij $+\gamma p 0 Z q j+\mathrm{Upj}_{\mathrm{Xpij}}+\mathrm{Uoj}+$ eij

Nota-se, contudo, que as variáveis randômicas devem pertencer ao nível mais baixo de análise, normalmente o nível individual, por permitir observar a influência do contexto sobre o comportamento de indivíduos, por exemplo. Se houver, por exemplo, a contribuição significativa do parâmetro estimado (razão t maior que 1,96 e redução da deviance em relação ao modelo anterior) da variável randômica "satisfação quanto à política de remunerações", o fato dele estar lotado em uma Unidade em que há, caracteristicamente, satisfação ou insatisfação quanto a tal política é fator impactante em seu desempenho. A análise do coeficiente de regressão indicará se tal influência se dará de forma positiva ou negativa na predição da variável critério associada. Maiores detalhes sobre como interpretar variáveis com efeito aleatório podem ser encontrados em Coelho Jr. (2009), Brandão (2008) e Ferrão (2003).

O último passo na modelagem multinível consiste na proposição de termos de interação, cuja equação de regressão é apresentada a seguir.

[5] Yij $=\gamma 00+\gamma p 0 X$ pij $+\gamma p 0 Z q j+\gamma p 0 Z$ ziX pij + UpjX pij + Uoj + eij

As variáveis em interação podem produzir efeitos diferentes de quando as mesmas são inseridas separadamente nos passos anteriores. A interação supõe que o efeito multiplicado das variáveis poderá exercer influência na predição da variável critério 
associada, e que tal efeito pode ter um impacto diferente se as mesmas variáveis da interação apresentarem contribuição significativa de forma isolada no modelo teórico. Caso se verifique, por exemplo, a ocorrência de uma interação estatisticamente significativa entre as variáveis "satisfação com a chefia" e "percepções compartilhadas de suporte à aprendizagem informal no trabalho por parte do chefe", sinaliza-se que o efeito da satisfação com a chefia é maior quando há percepções favoráveis compartilhadas do tipo de suporte à aprendizagem provido pela chefia. Os efeitos de interação entre níveis devem ser tratados com cuidado, pois são de difícil compreensão e análise. A interação pode ocorrer entre variáveis de um mesmo nível de análise (por exemplo, tempo de serviço x satisfação quanto ao ambiente de trabalho) ou entreníveis (como função $\mathrm{x}$ crenças compartilhadas sobre $\mathrm{O}$ clima organizacional). O tipo de contribuição dessa interação na predição da variável critério associada pode ser de forma positiva ou negativa, a depender da análise do coeficiente de regressão.

Caso sejam realizadas interações entre variáveis de um mesmo nível, tem-se $\gamma \mathrm{p} 0 \mathrm{X}$ pijX pij (Hox, 2002; Snijders e Bosker, 1999). Os coeficientes randômicos das variáveis de nível de contexto são independentes entre os grupos e possuem uma matriz de covariância constante com distribuição multivariada normal.

A seguir, apresenta-se um modelo final de equação multinível considerando a inserção de todos os passos anteriormente descritos. A variável critério considerada é desempenho individual. Tem-se:

[6] Yij $=\gamma 00+\gamma$ p0Xpij $+\gamma p 0 Z q j+\gamma p 0 Z q j X p i j+$ UpjXpij + Uoj + eij

Onde:

Yij=variável critério, como desempenho no trabalho do funcionário i na Unidade $\mathrm{j}$ da Empresa; $\gamma 00=$ intercepto, ou seja, valor médio do desempenho no trabalho dos funcionários sem a inclusão de nenhuma variável antecedente de explicação.

$\gamma \mathrm{p} 0$ Xpij=coeficiente de inclinação, representa o efeito $\gamma$ da variável explicativa $X$, de nível menos elevado; $\gamma \mathrm{p} 0 \mathrm{Zqj}=$ coeficiente de inclinação, representa o efeito $\gamma$ da variável explicativa $Z$, de nível de grupo;

$\gamma p 0 Z q j X p i j=$ coeficiente de inclinação, representa o efeito da interação ZX entre a variável explicativa $Z$, de nível menos elevado, e a variável explicativa $X$, do nível de grupo, indicando o efeito moderador que a variável de nível mais elevado $\mathrm{X}$ exerce sobre a relação entre a variável explicativa $Z$, de nível menos elevado, e a variável critério Yij;

UpjXpij=erro ou resíduo das inclinações de variáveis explicativas; eij=erro ou resíduo do nível menos elevado, por exemplo, parte do desempenho do funcionário não explicado pelo modelo;

Uoj=erro ou resíduo no nível de grupo.

Nessa sexta equação encontram-se todos os parâmetros a serem estimados e que permitirão uma análise empírica da estrutura de dados. Iniciou-se a análise com o modelo mais simples (vazio) e adicionaram-se, a cada passo, os vários tipos de parâmetro, inspecionando-se quais deles eram significativos e o quanto de variância era explicada. Verificar-se-á o quanto da variabilidade da variável resposta "desempenho" ocorrerá em virtude do tamanho do efeito de cada variável antecedente, analisada isoladamente ou em interação, com variáveis de mesmo nível e de níveis diferenciados.

\section{Conclusões}

O objetivo deste trabalho consistiu em apresentar possíveis contribuições da modelagem multinível no estudo de desempenho individual no trabalho. Foram detalhados todos os passos necessários à realização da modelagem multinível, exemplificando-se cada um deles com o uso de variáveis comumente investigadas em estudos em comportamento organizacional. Equações de regressão foram apresentadas considerando a influência de variáveis individuais (com parâmetro fixo e randômico), contextuais e termos de interação na predição de desempenho.

Teoriza-se que o fato dos indivíduos estarem num mesmo grupo social faz com que estejam submetidos a estímulos semelhantes, $\log$ compartilhem crenças e percepções. Esses estímulos exercem influência sobre os padrões de comportamento, e a atuação de cada indivíduo deve ser analisada a partir do efeito do contexto.

O estudo dos fenômenos multiníveis nas organizações relaciona-se a um complexo relacionamento entre métodos e estatísticas aliado ao desenvolvimento da teoria. É inegável o efeito que variáveis do contexto exercem sobre o comportamento individual, modelando-o e estabelecendo padrões esperados de conduta e modos de ser e de agir. Assim, acredita-se que a modelagem multinível é bastante relevante à explicação de variáveis de contexto na predição de desempenho individual no trabalho.

Um modelo multinível deve atender ao pressuposto da parcimônia, procurando explicar maior quantidade de variância da variável critério, considerando o menor número possível de variáveis antecedentes. Um problema multinível deve ser explicado por teorias multiníveis, atendendo-se ao 
princípio básico de que as variáveis sejam atribuídas corretamente em seu nível apropriado e quais efeitos diretos e de interação cross-level podem ser esperados. $\mathrm{O}(\mathrm{s})$ modelo(s) multinível(eis) investigado(s) deve(m) atender, também, aos pressupostos de normalidade e linearidade.

A discussão acerca da utilização da modelagem multinível no estudo de desempenho pode ser considerada uma importante contribuição deste trabalho. Ressalta-se que a análise empírica da contribuição significativa das interações cross-level na predição de desempenho somente será viabilizada por meio da modelagem multinível. Sua utilização deve ser realizada mais vezes nos estudos organizacionais. É preciso que as organizações investiguem o que pode estar associado ao desempenho no trabalho em uma perspectiva sistêmica. A análise multinível pode ser útil para fazer isto.

\section{Referências}

Afshartous, D. \& Leeuw, J. (2005). Prediction in multilevel models. Journal of Educational and Behavioral Statistics, 30(2), 109-139.

Bliese, P. D., Chan, D. \& Ployhart, R. E. (2007). Multilevel methods: future directions in measurement, longitudinal analyses, and nonnormal outcomes. Organizational Research Methods, 10(4), 551-563.

Brandão, H. P. (2008). Aprendizagem, contexto, competência $e$ desempenho: um estudo multinivel (Tese de Doutorado). Brasília: Universidade de Brasília Instituto de Psicologia.

Burke, M. J., Sapyr, S. A., Tesluk, P. E. \& SmithCrowe, K. (2002). General safety performance: a test of a grounded theoretical model. Personnel Psychology, 55, 429-457.

Coelho Jr., F. A. (2009). Suporte à aprendizagem, satisfação no trabalho e desempenho: um estudo multinivel (Tese de Doutorado). Brasília: Universidade de Brasília Instituto de Psicologia.

Derue, D. S. \& Morgeson, F. P. (2007). Stability and change in person-team and person-role fit over time: the effects of growth satisfaction, performance and self efficacy. Journal of Applied Psychology, 92, 1242-1253.

Ferrão, M. E. (2003). Introdução aos modelos de regressão multinivel em educação. Campinas: Komedi.
Hox, J. (2002). Multilevel analysis - techniques and applications. New Jersey: Lawrence Erlbaum Associates.

Klein, K. J. \& Kozlowski, S. W. J. (2000). Multilevel theory, research and methods in organizations. Foundations, extensions and new directions. São Francisco: Jossey-Bass.

Krull, J. L. \& MacKinnon, D. P. (1999). Multilevel mediation modeling in group-based intervention studies. Evaluation Review, 23(4), 418-444.

Lam, S. S. K. \& Schaubroek, J. (1999). Total quality management and performance appraisal: an experimental study of process versus results and group versus individual approaches. Journal of Organizational Behavior, 20, 445-457.

Lester, S. W., Meglino, B. M. \& Korsgaard, M. A. (2002). The antecedents and consequences of group potency: a longitudinal investigation of newly formedwork groups. Academy of Management Journal, 45, 352-368.

Maas, C. J. M. \& Hox, J. J. (2005). Sufficient sample sizes for multilevel modeling. Methodology, 1(3), 86-92.

Morgeson, F. P. \& Hoffmann, D. A. (1999). The structure and function of collective constructs: implications for multilevel research and theory development. Academy of Management Review, 8, 547558.

Nielsen, S. K. (2006). A multisource model of perceived organizational support and performance. (Dissertação de Mestrado). Knoxville: University of Tennessee.

Oliveira, M. R. (2007). Estudo longitudinal dos fatores associados ao desempenho escolar. (Tese de Doutorado). Brasília: Universidade de Brasília - Instituto de Psicologia.

Roberson, Q., Sturman, M. \& Simons, T. (2007). Does the measure of dispersion matter in multilevel research? A comparison of the relative performance of dispersion indexes. Organizational Research Methods, 10(4), 564-587.

Snijders, T. A. B. \& Bosker, R. J. (1999). Multilevel analysis: an introduction to Basic and Advanced Multilevel Modeling. Sage Publishers.

Sonnentag, S. \& Frese, M. (2002). Performance concepts and performance theory. Em S. Sonnentag (Ed.). Psychological Management of Individual Performance (pp.3-27). Great Britain: John Wiley \& Sons. 
Sobre os autores:

Francisco Antonio Coelho Junior é mestre e doutor em Psicologia Social, do Trabalho e Organizacional pelo Programa de Pós-Graduação em Psicologia Social, do Trabalho e Organizações da Universidade de Brasília; professor adjunto I do Departamento de Administração da Universidade de Brasília e professor do Programa de Pós-Graduação em Administração da Universidade de Brasília.

Jairo Eduardo Borges-Andrade possui Doutorado em Sistemas Instrucionais pela Flórida State University e PósDoutorado pela University of Sheffield. É professor titular do Departamento de Psicologia Social, do Trabalho e Organizacional da Universidade de Brasília. 